\section{Perceived magnitude of multiple electrocutaneous pulses}

\author{
R. M. SACHS, J. D. MILLER, and K. W. GRANT \\ Central Institute for the Deaf, St. Louis, Missouri 63110
}

The subjective magnitude of electrical stimulation has been studied extensively. Usually, the function relating the electrical stimulus (current) to magnitude estimates can be approximated by a straight line on loglog coordinates, with a slope between 1.8 (Ekman, Frankenhaeuser, Levander, \& Mellis, 1964, 1966; Sternbach \& Tursky, 1964) and 3.5 (e.g., S. S. Stevens, Carton, \& Shickman, 1958). The dynamic range of current that elicits sensations between threshold and pain varies inversely with this slope: high slopes correspond to small ranges $(6$ to $8 \mathrm{~dB})$ and low slopes correspond to larger dynamic ranges (up to $20 \mathrm{~dB}$ ). This inverse relation has been observed across many sensory modalities (e.g., Poulton, 1968; Rosenblith, 1959; Teghtsoonian, 1971, 1973), although there is disagreement over whether the relation is due to arbitrary choices of stimulus range or to true variations in stimulus dynamic range which produce the same range of sensation.

This study was motivated by the view that the configuration of stimulus (e.g., the type of current waveform) might influence growth of perceived magnitude. Sinusoidal and single-pulse waveforms have been employed in other magnitude studies and have been shown to produce pain with a small dynamic range. Gibson (1968) and Saunders (1974) developed a stimulus configuration for painless electrical stimulation using multiple pulses of current. This stimulus is "quantal." The Saunders scheme consists of bursts of alternating positive and negative pulses, each with a duration, $\tau$, of about $10 \mu \mathrm{sec}$ and a peak amplitude, $\mathrm{I}_{\text {peak }}$, of $10 \mathrm{~mA}$ (cf. Figure 1). Average intensity is changed by increasing or decreasing the number of such pulses within each burst. We hoped such stimulations would sesult in shallow loudness slopes and wide dynamic range in order to facilitate coding auditory information in the intensity dimension with a cutaneous stimulator.

In the present study, we have employed the method of cross-modality matching (CMM) to compare the perceived magnitude of electrical stimulation on the abdomen to that of a $500-\mathrm{Hz}$ tone presented by earphones. In this way, we could compare growth of sensation magnitude for the two modalities, since our ultimate goal is to substitute the skin for the ear as a communication channel. We could also infer the

R. M. Sachs' present address is: Network Performance Studies Department, Room 3D507, Bell Laboratories, Holmdel, New Jersey 07733. growth of the skin sensation knowing that the slope of the loudness/sound-pressure function for a $500-\mathrm{Hz}$ tone is about .6 (S. S. Stevens, 1966, 1968).

\section{METHOD}

\section{Subjects}

Three subjects, all with normal hearing, participated in this experiment. They were: R.M.S., male, age $33 ;$ K.W.G., male, age 23 ; and B.S.S., femaie, age 23 . The first two alternated as subject/experimenter.

\section{Stimuli}

Bipolar electrical pulses were delivered from a pulse generator circuit, originally designed by Saunders (1974), to the abdomen of the subject through single concentric electrodes. The electrode was located, depending on the subject, 8 to $12 \mathrm{~cm}$ to the right of the midline, about 1.3 to $3.7 \mathrm{~cm}$ above the navel (just below the rib cage). At the level of the electrode, waist circumferences were 86,72 , and $56 \mathrm{~cm}$ for subjects R.M.S., K.W.G., and B.S.S., respectively. The electrode was sterling silver with a raised center active area of $12 \mathrm{~mm}^{2}$ and a surrounding ground area of $260 \mathrm{~mm}^{2}$. The current waveform is shown in Figure 1. Current pulses (10-mA peak) were delivered at a burst rate, $R$, of $30 \mathrm{~Hz}$ to produce a sensation that was "flutter"-like. Perceived magnitude was varied by changing $N$, the number of pulses per burst, and $\tau$, the pulse duration. Each burst could contain from one to as many as 150 bipolar pulse pairs. The width of each pulse, $\tau$, was a parameter in the experiment, taking on values of 4.5 to $9 \mu \mathrm{sec}$, depending on subject and condition. Under these conditions, rms current ranged from .23 to $2.8 \mathrm{~mA}$ according to the equation:

$$
I_{\text {rms }}=I_{\text {reak }} \cdot \sqrt{2 N_{T} R}
$$

This equation is developed in Appendix 1.

A $500-\mathrm{Hz}$ tone was presented over earphones to the right ear of the subject. Intensity of this tone was under subject control through the use of a $40-\mathrm{dB}$ range attenuator callibrated in 1.5-dB steps. Tonal intensity was also under experimenter control with an additional attenuator in series so that the numbers on the subject's attenuator had no consistent reference.

\section{Procedure}

In the cross-modality matching procedure, the experimenter controlled electrical stimulus and the subject adjusted the auditory stimulus. During each trial, stimuli were repeated in a 5 -sec cycle: $.5-\mathrm{sec}$ electrical stimulation, $.5-\mathrm{sec}$ rest, $.5-\mathrm{sec}$ tone, and $3.5-\mathrm{sec}$ rest. Thus, the successive .5 -sec periods of electrocutaneous stimulation were separated by $4.5 \mathrm{sec}$. which was found in a preliminary experiment to be sufficiently long to prevent fading or adaptation

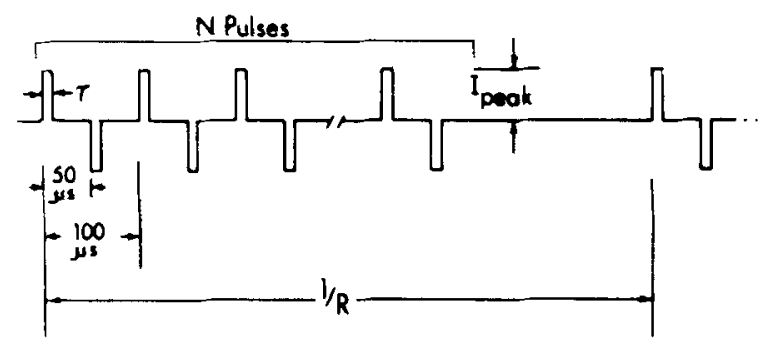

Figure 1. Current waveform delivered to the bipolar electrode indicating the variables $\tau$ (pulse width in microseconds), $N$ (pulses per burst), and $R$ (bursts per second). 
of the skin sensation from cycle to cycle. The task of the subject was to adjust the intensity of the tone so that its perceived magnitude matched that of the electrical stimulus.

On any given day, one subject was tested with the following overall procedure. First, the skin area receiving the electrode was cleaned with alcohol, then wetted with physiological saline before the electrode was strapped on. ${ }^{1}$ After the electrode wires were attached to the current pulse generator, the subject was "warmed up" with a mild level of current (typically $.6 \mathrm{~mA}$ rms, corresponding to a tonal CMM of 20 to $40 \mathrm{~dB} \mathrm{SL}$ ), using the same 5-sec cycling time as in the CMM experiment. This warm-up, lasting at least $5 \mathrm{~min}$, was followed by at least two or three practice trials using the CMM procedure. Then thresholds for both the tone and the electrocutaneous stimulation were obtained by an adjustment procedure. Tone levels were varied by the subject's attenuator. Current levels were adjusted by varying $\tau$, the duration of the current pulses, with a burst rate, $R$, of $30 \mathrm{~Hz}$, and a pulse number, $\mathrm{N}$, of 3. Then, an average of $20 \mathrm{CMM}$ adjustment trials were presented, with $\mathrm{N}$ and sometimes $\mathrm{T}$ being varied from trial to trial by the experimenter. Finally, current and tone thresholds were again obtained. Upon removal of the electrode, measurement of the electrode location was taken relative to the navel to insure the same stimulation site for a given subject.

\section{RESULTS}

\section{Cross-Modality Matching}

Data were taken with two pulse durations, $\tau$, for Subjects R.M.S. and K.W.G., and with one pulse duration for Subject B.S.S. Figure 2 shows the raw adjustments obtained with a burst rate, $R$, of $30 \mathrm{~Hz}$. The abscissa is $\mathbf{N}$, the number of pulses per burst, on a $\log$ scale, and the ordinate is sensation level of the matched tone in decibels. Each graph in this figure, representing one subject and pulse duration, contains form 58 to 125 adjustments (mean $=90$ ) obtained over 5 to 9 days. Each day is indicated by a different symbol. The experience in this CMM task prior to this data collection varied among subjects: R.M.S. and K.W.G. each practiced 15 days, and B.S.S. practiced 1 day. These differences in experience did not seem to affect variability in the data. A line of best fit is drawn through each set of data to minimize the mean square deviations in the y direction. The degree of correlation between rms current and tone levels ranged from $r=.40$ to $r=.91$. Similarly, the standard deviation about the best straight line ranged from \pm 14.1 to $6.7 \mathrm{~dB}$.

By reanalyzing the data, it was seen that the rather larger standard deviations in matched tonal level are actually much smaller, taken one day at a time. We noticed that, for any given subject and pulse duration, the best straight line describing the CMM data on one day was nearly parallel to those on other days. It was as if sensitivity for this electrical stimulus changed from day to day. By shifting a given day's data horizontally along the $\log \mathrm{N}$ axis, it was possible to reduce the standard deviations in the CMM data for each subject and pulse duration. The method by which these shifts were made is described in Appendix 2 . The results of this transformation are shown in Fig- ure 3. Each graph represents a different subject and pulse duration. The best straight lines shown in these five graphs are essentially the same as those from the corresponding unshifted data. The standard deviations about these lines, which were originally 7 to $14 \mathrm{~dB}$, are now 6 to $8 \mathrm{~dB}$. The correlation coefficients, originally, .40 to .91 , are now .92 to .93 . The unshifted data yielding the smallest scatter and standard deviations (for R.M.S. at $\tau=6 \mu \mathrm{sec}$ ) were not affected greatly by the shifting process. But the most variable unshifted data (for K.W.G. at $\tau=4.5 \mu \mathrm{sec}$ ) show a reduction in standard deviation by more than a factor of 2 when shifted. The correlation coefficients and standard deviations for all conditions and for both normal and shifted analyses are given in Table 1 .

If daily shifts in CMM functions are due to changes in sensitivity from day to day, then there ought to be a covariation of the shifts along the $\log \mathrm{N}$ axis used to construct Figure 3 and daily measures of pulse width at absolute threshold. However, the correlation between these two variables across all subjects and conditions is low $(r=.4) .^{2}$ One possible reason for this low correlation is that perceived magnitude may not depend strongly on what happens at threshold. Another very likely reason is that adjusting $\tau$ for threshold may be inappropriate: if $\mathrm{N}$ had been adjusted instead, the correlation might have been higher. This is because threshold pulse width is practically independent of pulse number, a finding described in the next section.

Another point of interest from Figures 2 and 3 concerns dynamic range: It was possible to produce electrocutaneous sensations that were matched to acoustic intensities from threshold to more than $70 \mathrm{~dB}$ above threshold. The corresponding range of rms current over which sensation grew was about $25 \mathrm{~dB}$ (due to changes in both $\tau$ and N.) However, on any given day the effective dynamic range for current might be reduced. For example, one day the current threshold might be elevated and, of course, the maximum pulse number $(\mathrm{N}=150)$ could not be exceeded (see Figure 2A, Day 5). Another day (especially for $\tau=9 \mu \mathrm{sec}$ ), current threshold might be so low that the minimum pulse number $(\mathrm{N}=1)$ would produce a suprathreshold sensation; yet sensations would also plateau for pulse numbers exceeding some moderate value (see Figure 2B, Day 3).

The primary quantity of interest in this study is the slope of the CMM function, which was relatively constant from day to day for a given subject and pulse width. The slopes of the best straight lines from Figure 3 are given in Table 2, column 3, as the ratio $\log$ sound pressure/log $N$. These slopes have been doubled to obtain another set of slopes in column 4 which relate sound pressure to rms current. The slope for all data combined is $3.0 \pm .1$ ( $95 \%$ confined limits). In the last column of this table are estimates of electrocutaneous sensation-magnitude slope, which 

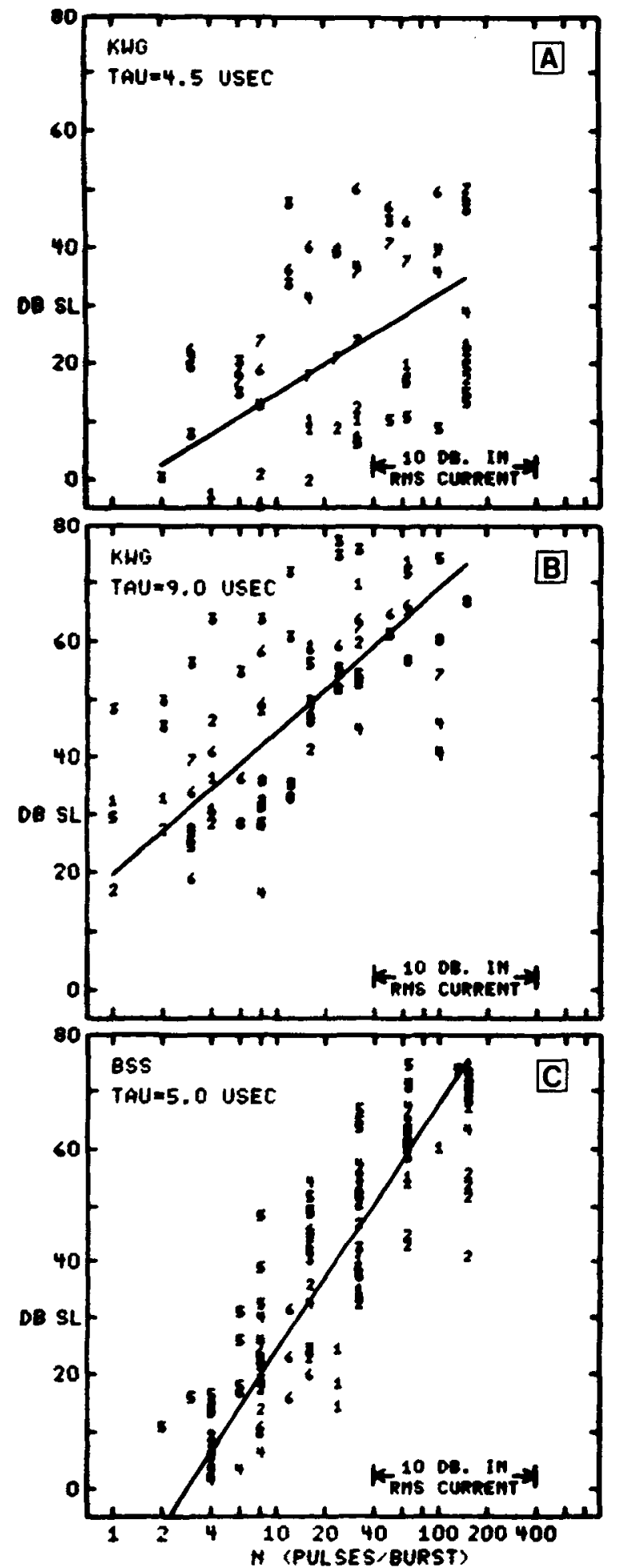

were obtained by multiplying the fourth-column slopes by .6, the slope relating log (magnitude estimate) to $\log$ (sound pressure) for a $500-\mathrm{Hz}$ tone (S. S. Stevens, 1966, 1968). The result is a mean slope of 1.8. In the discussion section, we compare this result with the slopes obtained by other investigators using electrocutaneous stimulation.

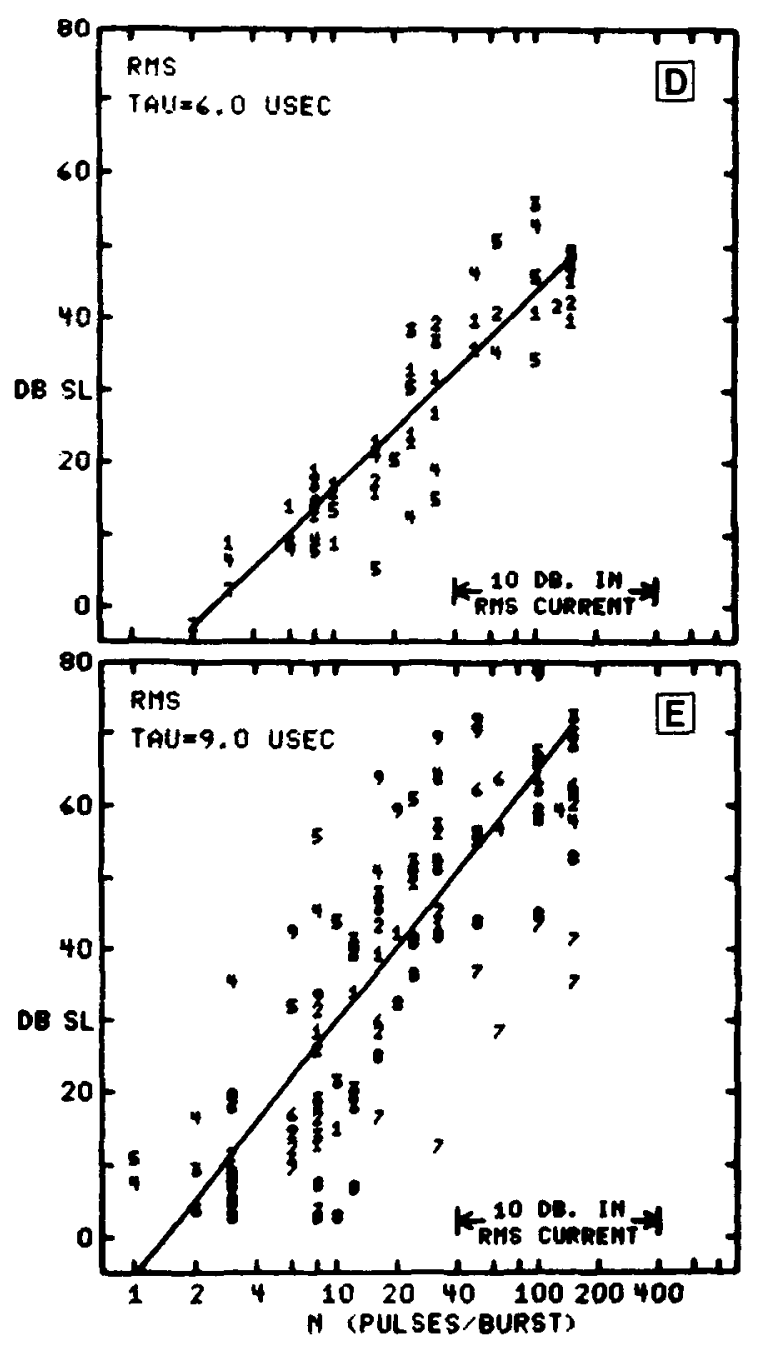

Figure 2. Cross-modality equal-loudness match of a $500-\mathrm{Hz}$ tone (in Db SL) for a given level of electrocutaneous stimulation (N pulses per burst). Each panel represents a different subject or pulse width, $\tau$. Each data point represents a single adjustment, its number indicating a different day in chronological order. Lines represent linear fits that minimize variation in matched tone level.

Another interesting aspect of our results concerns the relative contribution of pulse width, $\tau$, and pulse number per burst, $N$, to perceived magnitude. The data show, in a preliminary way, that sensation magnitude grows much faster with pulse width than with pulse number. From Figures 3A, 3B (Subject K.W.G.), 3D, and 3E (Subject R.M.S.), one can obtain 2-point estimates of the slope relating log pressure to $\log \tau$ at any N. This slope varies from 4 to 6.3 (8 to 12.6 in terms of rms current) for K.W.G., depending on N. For R.M.S., the corresponding slope varies from 2.4 to 5.6 (4.8 to 11.3). At an intermediate value of $N(16)$, the slope relating $\log$ pressure to $\log$ current (by varying $\tau$ ) is 9.5 for K.W.G. and 8.4 for R.M.S. In contrast, the same slope when varying $N$ is about 2.2 for K.W.G. and 3.0 for R.M.S. when averaged over the two values of $\tau$ (see Table 2). Thus, 

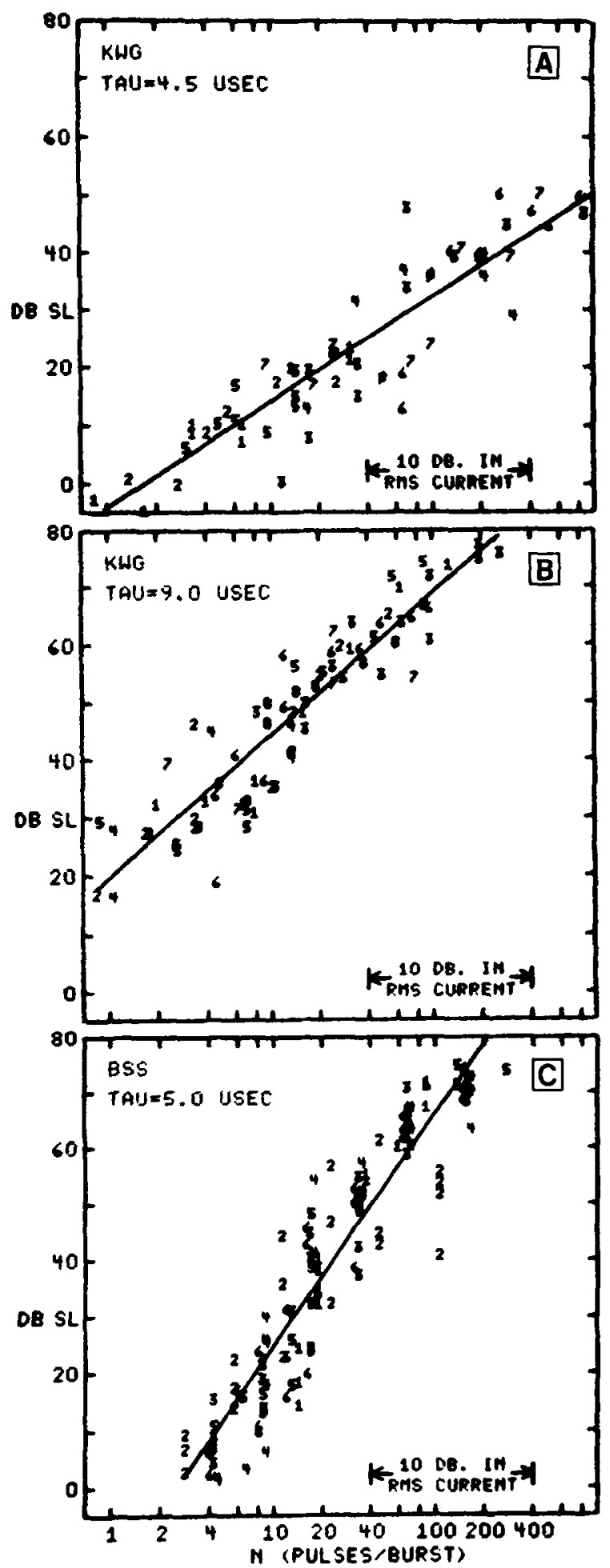

electrocutaneous sensation magnitude appears to grow three to four times faster with pulse width than with number of pulses per burst. ${ }^{3}$ This result is at odds with the notion that the only aspect of the stimulating waveform that contributes to sensation magnitude is rms current. (Recall that, for our pulsed stimulation, $I_{r m s}=I_{\text {peak }} \cdot \sqrt{2 \sqrt{\tau R} \text {. }}$ ) The notion that because $\mathrm{N}$ and $\tau$ contribute equally to rms current,

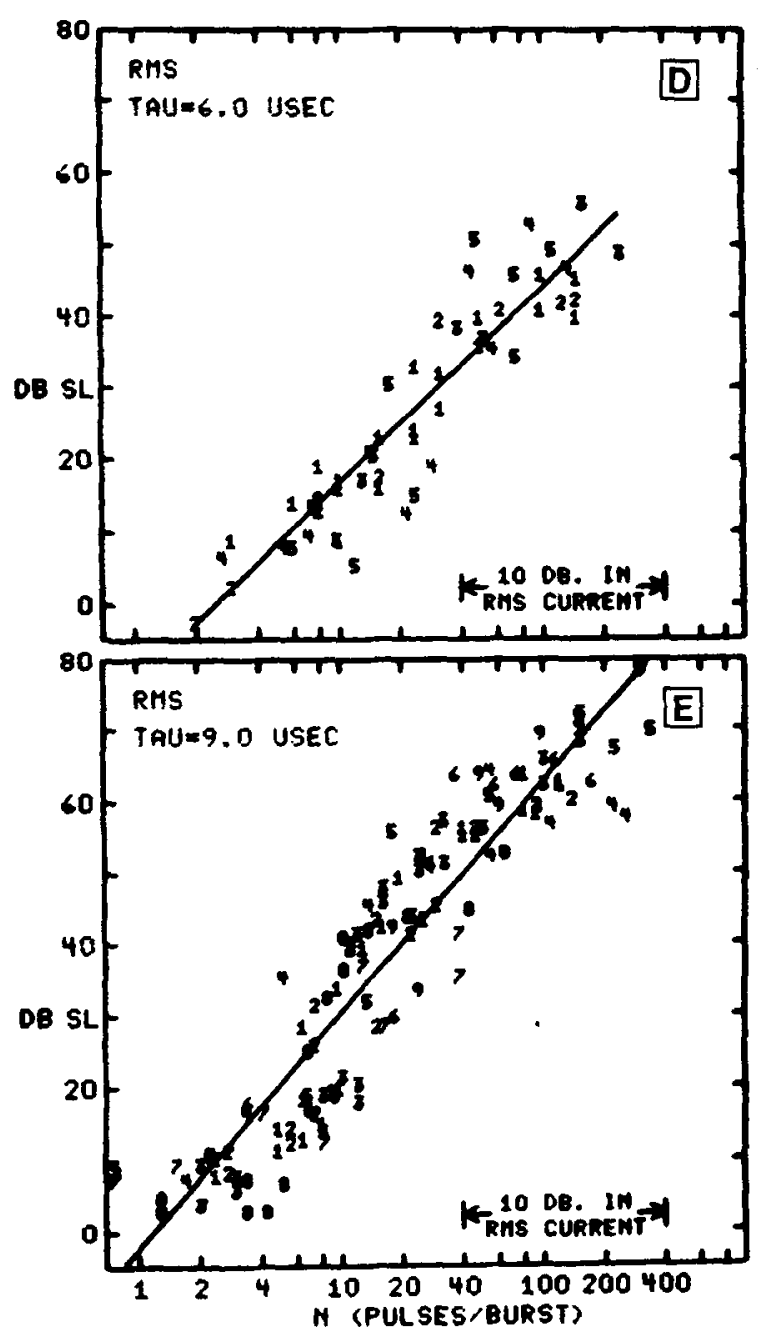

Figure 3. Same as Figure 2, except that data points have been shifted horizontally, an equal amount for a given day, so as to further reduce the variation in matched tone level, according to the method of Appendix 2.

they also contribute equally to loudness is not supported by our data.

\section{Trading of $\tau, \mathbf{N}$, and $\mathbf{R}$}

In a separate experiment, by examining directly the tradability of $\tau, N$, and $R$ in producing signals that are just detectable and signals that have equal sensation magnitude, we tested the hypothesis that loudness is based only on rms current. Figure 4 shows how $\tau, N$, and $R$ trade at threshold for two subjects. Stimuli of $.5 \mathrm{sec}$ duration were presented every $5 \mathrm{sec}$. When $\tau$ is adjusted for threshold, there is little dependence on $N$ or $R$ : a reduction in $\tau$ of only $25 \%$ is required for a 10 -fold increase in either $\mathrm{N}$ or $\mathrm{R}$ (a trade of $1: 8$ ).

Next, we measured the tradability of $N$ and $R$ at fixed $\tau$ for suprathreshold levels in a tactile loudnessbalance task. In this case, we presented .5 -sec stimuli every $3 \mathrm{sec}$, so that a standard tactile stimulus alternated with one in which the subject could adjust $\mathbf{N}$. 
Table 1

Correlation Coefficients and Standard Deviations

in Matched Tone Levels for CMM Experiment in Which Pulse Number is Varied

\begin{tabular}{|c|c|c|c|c|c|c|}
\hline \multirow[b]{2}{*}{ Subject } & \multirow[b]{2}{*}{$\tau^{*}$} & \multirow{2}{*}{$\begin{array}{l}\text { Number } \\
\text { of Trials }\end{array}$} & \multicolumn{2}{|c|}{$\mathrm{r}$} & \multicolumn{2}{|c|}{$\mathrm{SD}^{* *}$} \\
\hline & & & $\mathrm{N}$ & S & $\mathrm{N}$ & $S$ \\
\hline K.W.G. & 4.5 & 65 & .40 & .92 & 14.1 & 6.2 \\
\hline K.W.G. & 9.0 & 77 & .63 & .92 & 12.4 & 6.3 \\
\hline B.S.S. & 5.0 & 123 & .88 & .93 & 10.4 & 7.9 \\
\hline R.M.S. & 6.0 & 58 & .91 & .92 & 6.7 & 6.1 \\
\hline R.M.S. & 9.0 & 125 & .81 & .93 & 12.6 & 7.9 \\
\hline Combined & & 448 & .64 & .92 & 16.3 & 8.1 \\
\hline
\end{tabular}

Note $-N=$ normal $S=$ shifted.

*In microseconds. **In decibels.

Table 2

Slopes of Cross-Modality Matching Functions (Shifted Data) Relating Sound Pressure (P) to Pulse Number $(N)$ and Current $\left(\mathbf{I}_{\mathrm{rms}}\right) \pm 95 \%$ Confidence Limits $(\mathrm{CL})$

\begin{tabular}{lccccc} 
Subject & \multirow{2}{*}{$\tau^{*}$} & $\begin{array}{l}\log \mathrm{P} / \\
\log \mathrm{N}\end{array}$ & \multicolumn{2}{l}{$\log \mathrm{P} / \log \mathrm{I}_{\mathrm{rms}}$} & $\log \mathrm{ME} /$ \\
\hline K.W.G. & 4.5 & .91 & 1.82 & .20 & 1.10 \\
K.W.G. & 9.0 & 1.22 & 2.47 & .24 & 1.46 \\
B.S.S. & 5.0 & 2.07 & 4.14 & .29 & 2.48 \\
R.M.S. & 6.0 & 1.37 & 2.74 & .30 & 1.64 \\
R.M.S. & 9.0 & 1.60 & 3.26 & .23 & 1.93 \\
Combined & & 1.49 & 2.98 & .12 & 1.79 \\
\hline
\end{tabular}

Note-Slope of magnitude estimate (ME) vs. current is obtained by multiplying $P$ vs. $I_{r m s}$ slope by .6. *In microseconds.

The standard and variable burst rates were also different, in general. Results for two subjects are shown in Figure 5 in graphs of $\log \mathrm{N}$ vs. Log R. The standard $R$ was fixed at $30 \mathrm{~Hz}$. The standard $N$ was either 16 or 64 pulses/burst. The data conform fairly well to a slope of -1 , implying a $1: 1$ trade between $N$ and $R$. This trade begins to break down for lower burst rates, suggesting that loudness is based on individual bursts of pulses if the bursts occur far enough apart in time.

There is an important consequence to our observation of $1: 1$ trading between $\mathrm{N}$ and $\mathrm{R}$. If one chooses to change pitch only by varying $R$, one must simultaneously vary $\mathrm{N}$ to avoid changes in sensation magnitude.

\section{DISCUSSION}

\section{Exponent for Electrocutaneous Loudness}

Our average estimate of the slope of the function relating electrocutaneous magnitude to rms current is about 1.8 when pulse number, $\mathrm{N}$, is varied. This slope, which estimates the power function exponent for the magnitude of electrocutaneous sensation, may seem very shallow compared with the classical results of S. S. Stevens and his co-workers at the Harvard Laboratory of Psychophysics (J. C. Stevens, Mack, \& S. S. Stevens, 1960; S. S. Stevens, 1959;
S. S. Stevens et al., 1958). Using finger-cup electrodes and $60-\mathrm{Hz}$ sinusoidal current that varied in amplitude, these researchers obtained slopes of about 3.5. Our shallower slopes suggest a more practical scheme for coding intensive information due to the resulting wider dynamic range of current that can be employed. However, we cannot necessarily attribute this slope difference to the particular kind of current pulse stimulation, body location, or electrode configuration we used. Cross, Tursky, and Lodge (1975), Sternbach and Tursky (1964), and others, using concentric electrodes on the dorsal forearm and sinusoidal amplitude-varying current, obtained exponents of 1.8 to 2.3. This suggests that the use of sinusoidal current is not the reason for large exponents. More important, Sternbach and Tursky (1964) have replicated the Harvard experiments in all respects (method, location, electrode configuration, and type of stimulation), with a resulting exponent averaging 1.9 . They suggest that the different outcome is due to differences in subjects. Furthermore, varying pulse number is not the only way to obtain shallow slopes with pulsed stimulation. Babkoff (1978) obtained magnitude estimation slopes for single, variableamplitude current pulses that ranged from 1.4 to .9 as pulse width changed from 100 to $6,400 \mu \mathrm{sec}$.

Psychophysical method can also be an important difference in comparing studies. Those experiments in which cross-modality matching was used (e.g., Cross et al., 1975, and S. S. Stevens, 1959) were counterbalanced in such a way as to minimize regression bias. Our investigation was not balanced, in that subjects varied the stimulus only in the auditory
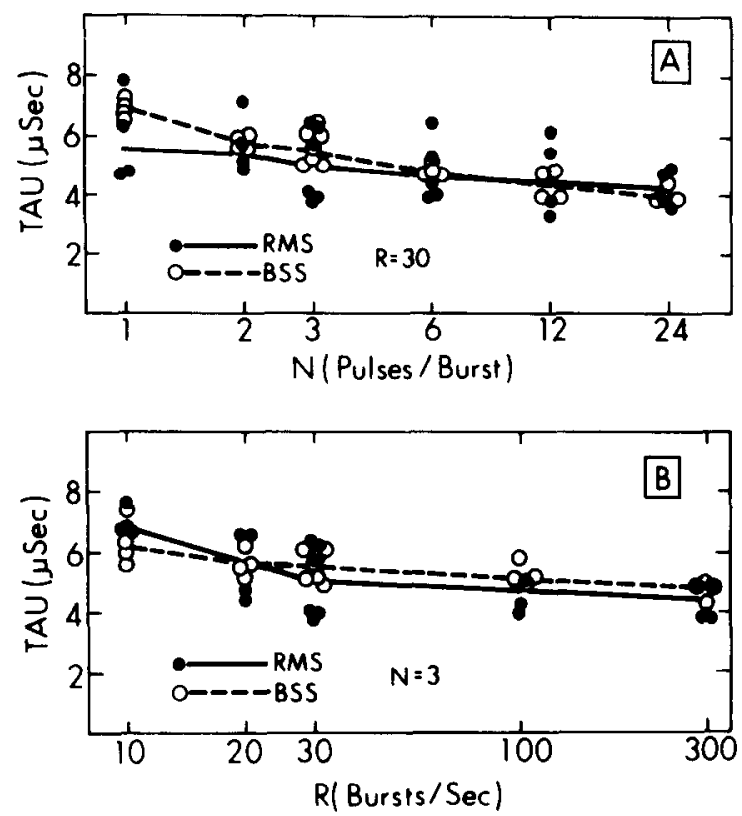

Figure 4. Tradability of $\tau, N$, and $R$ at threshold for two subjects. Each data point represents a single adjustment of tau as a function of either $R$ (panel $A$ ) or $N$ (panel B). Lines are drawn through median values. 


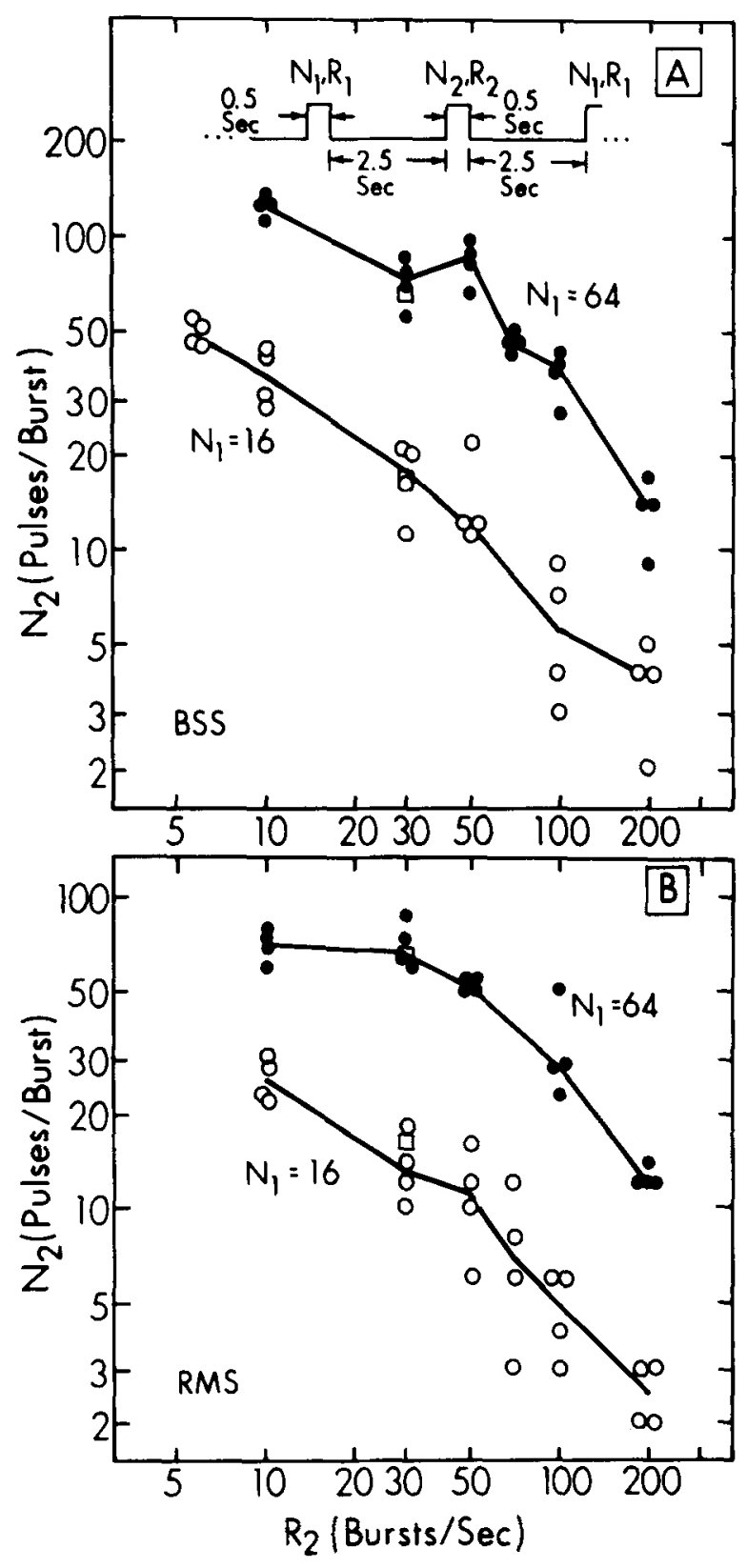

Figure 5. Tradability of $\mathbf{N}$ and $\mathbf{R}$ for suprathreshold electrocutaneous loudness balance. Each data point represents one adjustment of the variable $N_{2}$ for a given $R_{2}$ to match a standard pulse train consisting of $N_{1}$ (16 or 64 pulses) and $R_{1}$ (30 bursts/ sec). Each panel is a different subject. Time course of alternating stimuli is indicated in panel $A$ top. Lines are drawn through median values. Tau $=6 \mu \mathrm{sec}$ for B.S.S. $\left(N_{1}=16,64\right)$ and R.M.S. $\left(N_{1}=16\right)$. Tau $=9 \mu \sec$ for R.M.S. $\left(N_{1}=64\right)$.

modality. The result is that our slopes are underestimated due to regression bias. Thus, it appears possible that the use of pulse-number modulation with concentric electrodes on the abdomen does not produce sensation-magnitude functions any different from those obtained with sinusoidal current or singlepulse stimulation at other body sites or with other electrode configurations. Claims of differences could best be evaluated in a balanced experiment with many types of stimulation using the same subjects and method.

A statement should also be made about the number of subjects we have used in our cross-modality matching experiment. Most studies of perceived magnitude use a larger subject base, typically 10 or more, to derive an estimate of the loudness exponent. Thus, our combined cross-modality matching slope of $\mathbf{3 . 0}$ may have changed to some new value outside the \pm .1 range (see Table 1) if more subjects had been used. However, we chose in this study to emphasize the within-subject variability one obtained in this procedure. The characteristics of this variability must be understood if one is to properly fit a sensory substitution device on individuals. Hence, we observe that when large variability exists, it is predominantly due to day-to-day changes in absolute current, yielding a given tonal match rather than the rate of growth of the tonal match as current increases.

\section{Interaction of $\tau, N$, and $R$}

In our evaluation of tradability of $\tau, \mathbf{N}$, and $\mathbf{R}$, we noted that increasing $R$ above $30 \mathrm{~Hz}$ could be offset by a proportional decrease in $\mathrm{N}$ to maintain sensory magnitude. However, an increase in $\mathbf{R}$ or $\mathbf{N}$ by a factor of 10 could be offset by only a very slight decrease $(25 \%)$ in pulse width, $\tau$, at threshold. This latter result is consistent with that of Saunders (1974), who found that pulse width at threshold for single-pulse bursts was essentially unchanged for burst rates of 60 and $200 \mathrm{~Hz}$. The relative inability of pulse number to affect threshold has been documented by Gibson (1968) and Rollman (1974) for pulse numbers exceeding 3 or 4 . In these cases, the trade was between pulse number and pulse amplitude (rather than duration), but in Rollman's experiment pulse width was $100 \mu \mathrm{sec}$, and, at this and shorter durations, threshold is affected equally by amplitude and duration (Rollman, 1974; Saunders, 1974). It is interesting that Rothenburg and Verrillo (1976), using mechanical vibration, also concluded that threshold displacement amplitude was little affected by frequency if trains of single narrow pulses were used instead of sine-wave stimulation. Our result is similar to theirs if one considers current-pulse amplitude analogous to vibration displacement.

\section{Coding Intensity Information}

Another important issue concerns the coding of information by varying the intensity of electrocutaneous stimulation. The most direct way to evaluate pulse-number coding of intensity is to use the method of absolute identification with a set of "equally spaced" and easily discriminable stimuli (Garner \& Hake, 1951). In a short experiment, we performed this task using two subjects, presenting stimuli containing random values of $\mathrm{N}$ and using .5 -sec-duration $30-\mathrm{Hz}$ bursts repeated every $5 \mathrm{sec}$. Under the best of conditions (given knowledge of the stimulus set, un- 
limited time to respond, and trial-by-trial feedback), both observers could identify three widely spaced stimuli perfectly. When five or seven stimulus choices were given, the observers did not improve their information transfer significantly, even though the alternatives could always be discriminated pairwise. This result is consistent with other studies of tactile information coding. Hawkes (1961) measured identification of electrical stimulation by varying the amplitude of a sinusoidal current using a fingertip electrode. He found that subjects could absolutely identify (with no errors) only two steps throughout the usable dynamic range of current. He stated that three alternatives might yield $100 \%$ identification performance with extensive practice. The only other study of tactile information coding of intensity known to us concerns vibratory, not electrical, stimulation. Geldard (1957) chose to limit the number of absolutely identifiable steps to three within the range of 20 to $400 \mu$ on the ventral thorax.

\section{Intensity Coding in Tactile Aids for the Deaf}

Electrocutaneous stimulation has been used by several investigators as a sensory substitution for the deaf. Both Saunders (1974) and Sparks, Kuhl, Edmonds, and Gray (1978) used spectrally based electrode arrays on the abdomen, and both used pulsed stimulation. In Saunders' scheme, the energy in each band of audio frequencies is used to vary the pulse number, $\mathrm{N}$, delivered to a given electrode. Thus, auditory intensity is mapped into tactile intensity by varying $\mathrm{N}$. Sparks chose to code intensity spatially: Each frequency region is represented by a column of eight electrodes; electrodes higher up in the column are stimulated for greater auditory intensity. In a nonspectral patterning scheme, Scott and De Filippo (1977) used electrocutaneous stimulation with up to three electrodes on the hand. Like Saunders, Scott coded intensity with the $\mathrm{N}$ variable. In all of the above schemes, the burst rate was held constant (at either 100 or $160 \mathrm{~Hz}$ ), thus avoiding the problem of having both $\mathrm{N}$ and $\mathrm{R}$ affect sensory magnitude. It is not clear which method of coding intensity (spatially or with $\mathrm{N}$ ) can provide more information to the user, although the spatial method appears to have the potential advantage, given enough distance between electrodes.

\section{Summary and Conclusions}

A scheme to code sensory magnitude with pulsed electrocutaneous stimulation was used with a single concentric electrode by changing the number of current pulses per burst of pulses, as suggested by Saunders (1974). Using a burst rate of $30 \mathrm{~Hz}$ to achieve a flutterlike sensation, sensory magnitude was evaluated by varying the magnitude of a $500-\mathrm{Hz}$ tone to match the perceived electrocutaneous magnitude. The inferred relation for this kind of stimulation sensation is that electrocutaneous magnitude grows as the 1.8 power of rms current. It was observed that much of the variability in the data for matched tonal level (as much as $\pm 14 \mathrm{~dB}$ ) could be accounted for by day-to-day variations in current sensitivity. When this variability is removed through statistical manipulation of the data, all standard deviations are reduced to about $\pm 7 \mathrm{~dB}$ and correlations are in excess of .91 . In spite of our efforts, we were unable to discover a method to eliminate the variations as they actually occurred. While the slope of the matching functions remained relatively stable from day to day, the maximum match, the minimum match, and the difference between the two all varied over ranges of nearly $30 \mathrm{~dB}$. These large changes were apparent to the observer even before the matching task was begun.

In a separate experiment, the tradability of pulse width, $\tau$, pulse number per burst, $N$, and burst rate, $R$, was examined. As $R$ was increased 10 -fold, threshold or suprathreshold sensations were held constant by either a proportional 10 -fold decrease in $\mathrm{N}$ or only a slight $(25 \%)$ decrease in $\tau$.

It was observed that, like other forms of electroand vibrotactile stimulation, the use of pulse number, $\mathrm{N}$, to code intensity would result in the use of only three stimulus levels that could be absolutely identified. The chief advantage of this coding scheme is its ability to provide a wide range of sensations without evoking pain.

\section{REFERENCES}

BАвкоғғ, H. Electrocutaneous psychophysical input-output functions and temporal integration. Perception \& Psychophysics, $1978,23,251-257$.

Cross, D. V., Tursky, B., \& Lodie, M. The role of regression and range effects in determination of the power function for electric shock. Perception \& Psychophvsics, 1975. 18, 9-14.

Ekman, G., Frankenhaeuser, M., Levander, S., \& Mellis, I. Scales of unpleasantness of electrical stimulation. Scandinavian Journal of Psychology. 1964, 5, 257-261.

Ekman, G.. Frankenhafuser. M., Levander, S., \& Mellis, I. The influence of intensity and duration of electrical stimulation on subjective variables. Scandinavian Journal of Psvchologv, 1966. 7, 58-64.

Garner. W. R., Hake, H. W. The amount of information in absolute judgment. Psychological Review, 1951, 58, 446-459.

Geldard, F. A. Adventures in tactile literacy. American Psychologist, 1957, 12, 115-124.

Gibson, R. H. Electrical stimulation of pain and touch. In D. R. Kenshalo (Ed.), The skin senses. Springfield: Thomas, 1968.

Hawkes, G. R. An evaluation of the magnitude estimation technique. Journal of Psychologv, 1960, 50, 303-313.

Hawkes, G. R. Cutaneous discrimination of electrical intensity. American Journal of Psychology, 1961, 74, 45-53.

Poulton. E. C. The new psychophysics: Six models for magnitude estimation. Psychological Bulletin, 1968, 69, 1-19.

Rollman, G. B. Electrocutaneous stimulation. In F. A. Geldard (Ed.), Conference on Cutaneous Communication Systems and Devices. Austin, Tex: Psychonomic Society, 1974

Rosenhitu, W. A. Sensory performance of organisms. Reviews of Modern Phusics, 1959. 31, 485-491.

Rothenherc; M.. \& Veril,o, R. T. Vibrotactile frequency for encoding speech parameters, fournal of the Acoustical Societv of America, 1976, 59, 569 
Saunders, F. A. Electrocutaneous displays. In F. A. Geldard (Ed.), Conference on Curaneous Communication Systems and Devices. Austin, Tex: Psychonomic Society, 1974.

SaUnders. F. A. Recommended procedures for electrocutaneous displays. In T. Habrecht \& J. B. Reswick (Eds.). Functional electrical stimulation. New York: Dekker, 1977.

Scotr. B. L., \& De Filippo, C. L. Progress in the development of a tactile aid for the deaf. Journal of the Acoustical Society of America, 1977, 62, S76.

Sparks, D. W., Kuhl, P. K., Edmonds, A. E., \& Gray, G. P. Investigating the MESA (Multipoint Electrotactile Speech Aid): The transmission of segmental features of speech. Journal of the Acoustical Society of America, 1978, 63, 246-257.

Sternbach, R. A., \& Tursky, B. On the psychophysical power function in electric shock. Psychonomic Science, 1964, 1, 217-218.

Stevens. J. C., Mack, J. D.. \& Stevens. S. S. Growth of sensation on seven continua as measured by force of handgrip. Journal of Experimental Psychology, 1960, 59, 60-67.

Stevens, S. S. Cross-modality validation of subjective scales for loudness, vibration, and electric shock. Journal of Experimental Psychology, 1959, 57, 201-209.

Stevens, S. S. Power-group transformations under glare, masking and recruitment. Journal of the Acoustical Society of America, 1966, 39, 725-735.

Stevens, S. S. Tactile vibration: Change of exponent with frequency. Perception \& Psychophysics, 1968, 3, 223-228.

Stevens, S. S., Carton, A. S., \& Shickman, G. M. A scale of apparent intensity of electric shock. Journal of Experimental Psvchologv, 1958, 56, 328-334.

Tegitsoonian, R. On the exponent in Stevens' law and the constant in Ekman's law. Psychological Review, 1971, 78, 71-80.

Teghtsoonian, R. Range effects in psychophysical scaling and a revision of Stevens' law. American Journal of Psychologv, $1973,86,3-27$.

\section{NOTES}

1. The use of physiological saline follows the recommendation of Saunders (1974). In our experience, skin moisture is maintained under the electrode throughout the experimental session, in agreement with Saunders (1977). Thus, we would not expect current sensitivity to change during a session due to a change in salt concentration.

2. The correlation was between $\mathrm{Xshift}_{\mathrm{i}}$, the $\mathrm{i}^{\text {th }}$ day's shifted distance along the $\log \mathrm{N}$ axis, and $\log \tau_{\mathrm{oj}}$, the $\log$ of the $\mathrm{i}^{\text {th }}$ day's threshold width. Each $\tau_{\mathrm{oi}}$ was first normalized for a given subject's mean threshold, so as to obtain a correlation independent of this factor.

3. From Figure 3, one can also obtain a two-point estimate of how $\tau$ interacts with $\mathrm{N}$ to produce a constant cross-modality match. The result is variable, but nevertheless illustrates how much more sensitive one is to changes in $\tau$. For example, at an intermediate tone adjustment of $35 \mathrm{~dB} \mathrm{SL}$, a doubling of $\tau$ for Subject K.W.G. was offset by a decrease in $N$ from 150 to $4(37: 1)$. For Subject R.M.S., a similar computation results in a reduction in $\mathrm{N}$ of $7: 1$ for an effective doubling of $\tau$.

\section{APPENDIX 1}

\section{Derivation of rms Current}

The current waveform delivered to the bipolar electrode is depicted in Figure 1. Biphasic current pulses have pulse width $\tau$ and repeat every .1 msec until a burst of $\mathrm{N}$ pulses have occurred. The bursts are repeated every $1 / R$ sec. From the definition of root mean square,

$$
\begin{aligned}
I_{\text {rms }} & =\sqrt{\frac{1}{T} \int_{0}^{T} I^{2}(t) d t} \\
& =\left[R \int_{0}^{1 / R} I^{2}(t) d t\right]^{1 / 2}
\end{aligned}
$$

$$
\begin{aligned}
& =\left[R \cdot 2 N \int_{0}^{\tau} I_{\text {peak }}^{2} d t\right]^{1 / 2} \\
& =\left[R \cdot 2 N \cdot I_{\text {peak }}^{2} \cdot \tau\right]^{1 / 2}=I_{\text {peak }} \sqrt{2 N_{\tau} R} .
\end{aligned}
$$

where $\mathrm{T}=$ waveform period. In our CMM expcriments. $\mathrm{R}=30 \mathrm{~Hz} . \mathrm{N}=110150), \tau=4.5109 \mu \mathrm{sec}$, and $\mathrm{I}_{\text {peak }}=$ $10 \mathrm{~mA}$. Thus, $1_{\mathrm{rm}}$ lies in the range from $.164102 .85 \mathrm{~mA}$.

\section{APPENDIX 2 \\ Method of Data Shift}

Data points in Figure 2 have been shifted horizontally in such a way as to reduce the mean square deviations in the vertical $(y)$ direction. This shifting was accomplished in the following manner:

(1) For the $i^{\text {th }}$ day's set of data, a best linear fit is obtained (minimizing the standard deviations about the line in the $\mathrm{y}$ direction). This line is described by its slope and average $x$ and $y$ values: slope $e_{i}, \bar{X}_{i}, \bar{Y}_{i}$. Note $\bar{Y}_{j}$ is expressed in $\mathrm{dB} S \mathrm{SL}$, whereas $\bar{X}_{i}$ is expressed in $\log N$ units.

(2) The median slope, slope $m$, is calculated from the $n$ individual slopes.

(3) An $x$-intercept, $X_{i n t}$, is calculated for the $i^{\text {th }}$ line using the median slope:

$$
\text { Xint }_{i}=\bar{X}_{i}-\frac{\bar{Y}_{i}}{\text { slope }_{m}} .
$$

(4) Each line and its corresponding data points are shifted by the difference between $\mathrm{Xint}_{\mathrm{i}}$ and the mean $\mathrm{r}$-intercept.

$$
X \operatorname{shift}_{i}=\overline{X i n t}-X i n t_{i}=\frac{\sum_{1}^{n} X i n t_{i}}{n}-X i n t_{i} .
$$

(5) A new hest linear fit is calculated from the shifted data. The procedure is illustrated in Figure A1.

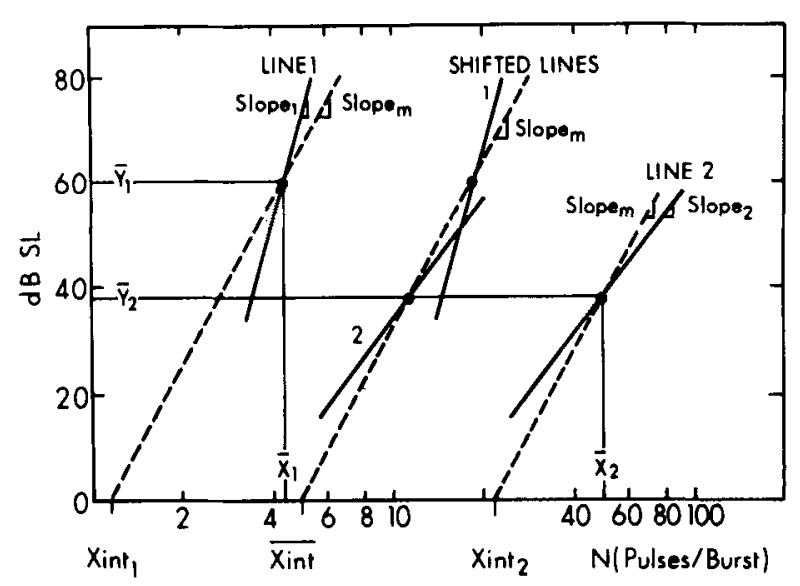

Figure A1. Illustration of method for shifting cross-modality matching data horizontally so as to reduce standard deviation in matched tone level. See Appendix 2 for details.

(Received for publication November 3, 1979; accepted June 20, 1980.) 